\title{
Stability of Stochastic Differential Switching Systems with Time-Delay and Impulsive Effects
}

\author{
Zhuang Fang $(\mathbb{D}$, Xiaozhong Huang, and Xuegang Tan \\ School of Science, Hubei University for Nationalities, Enshi 445000, China \\ Correspondence should be addressed to Xuegang Tan; redloner_tan@hotmail.com
}

Received 18 November 2017; Accepted 29 March 2018; Published 7 May 2018

Academic Editor: Sabri Arik

Copyright (C) 2018 Zhuang Fang et al. This is an open access article distributed under the Creative Commons Attribution License, which permits unrestricted use, distribution, and reproduction in any medium, provided the original work is properly cited.

\begin{abstract}
This paper studies the stability of hybrid impulsive and switching stochastic neural networks. First, a new type of switching signal is constructed. The stochastic differential switching systems are steerable under the work of the switching signals. Then, using switching Lyapunov function approach, Itô formula, and generalized Halanay's inequality, some global asymptotical and global exponential stability criteria are derived. These criteria improve the existing results on hybrid systems without noises. An example is given to demonstrate the effectiveness of the results.
\end{abstract}

\section{Introduction}

A switching system consists of several subsystems and a rule that orchestrates the switching among them. Switching plays an important role in many fields, such as optimal control, electrical circuit control, and other practical applications $[1$, 2]. However, switching is also a main source of instability and often deteriorates system performances. It is important and necessary to study the stability of switching systems [3].

Linear switching systems are primary and the earliest researched switching systems [4]. The model can be written as

$$
\dot{x}=A_{i_{k}} x(t), \quad t \in\left[t_{k}, t_{k+1}\right), \quad k=0,1, \ldots,
$$

where $A_{i_{k}} \in R^{n \times n}, x(t) \in R^{n}$ is the trivial solution. Switching signal $\sigma: R^{+} \rightarrow\{1,2, \ldots, N\}$ is represented by $\left\{i_{k}\right\}$ according to $\left[t_{k}, t_{k+1}\right) \rightarrow i_{k} \in\{1,2, \ldots, N\}$. In general, switching signal $\sigma$ is a piecewise constant function. In [4], based on a valid Lyapunov function for each subsystem of (1) in some subregion of $R^{n}$, the authors presented a state dependent switching rule to stabilize linear system (1) if the systems exist in a Hurwitz linear convex combination, $H=\sum_{i=1}^{N} \beta_{i} A_{i}$, $\sum_{i=1}^{N} \beta_{i}=1, \beta_{i} \in(0,1)$. Kim et al. studied the stability of a finite number of linear ordinary differential equation systems with time delay [5].
Recently, more and more researchers focus on the nonlinear switching ordinary differential equation systems (see, e.g., [6-12] and references therein). The general nonlinear form is given as follows:

$$
\dot{x}=f_{i_{k}}(x(t)), \quad t \in\left[t_{k}, t_{k+1}\right), \quad k=0,1, \ldots
$$

Fruitful results on the stability of the switching nonlinear systems are reported. For instance, Wang et al. discussed the finite-time stability problem for a class of impulsive switching systems with nonlinear perturbations and provided a sufficient condition for finite-time stability of perturbed switching systems [7]. In [8], some stability results were given by an average dwell-time approach for nonlinear switching systems. Hu and Michel analyzed a dwell-time scheme for local asymptotic stability of nonlinear switching systems with the activation time being used as the dwell time [10]. Lee and Lim considered continuous-time nonlinear switching systems and provided the stability results for nonlinear timevarying polytypic systems [11].

In addition, delay is also considered in many models. In $[13,14]$, the authors established several criteria on exponential stability for nonlinear systems with time delay by using multiple Lyapunov function technique and a dwell-time approach. Recently, $\mathrm{Pu}$ et al. considered the problem of stability of switching delay systems in $[1,15]$. 
As is known, practical systems are always affected by external disturbances, which may degrade the system performances. The chief problem that needs solving is how to eliminate the negative effects of the external disturbances as far as possible. With the development of stochastic analysis theory, stochastic systems are proposed to solve this kind of problems. These stochastic systems are adequate mathematical models for many processes and phenomena [16, 17]. Since the stochastic models have much more substance and are also important for many branches of science and engineering $[18,19]$, many researchers studied the stability of stochastic systems (see [20-25] and references therein). For instance, $\mathrm{Li}$ et al. [20] considered the stability of a class of impulsive stochastic neural networks with delays by resorting new integral inequalities and using the properties of spectral radius of nonnegative matrix. In their methods, some global $p$-exponential stability criteria of periodic solution for impulsive stochastic neural networks with delays were given. Furthermore, in [21-24], stability of some kinds of stochastic systems were discussed and some $p$ th moment stability criteria were obtained for impulsive stochastic systems with Markovian switching.

Although the stability of stochastic systems stirred some initial research interest, few works discussed the stability of nonlinear switching differential systems including stochastic, time-delay, and impulsive effects simultaneously. The stability analysis is more involved, since time-delay, impulsive, and stochastic effects are all considered in switching system. Many criteria of stability for differential dynamical systems (such as the systems of paper [26-32]) may be ineffective.

To stabilize the systems, it is necessary to construct a suitable switching signal to reduce the negative effects of time delay and external disturbances. It is no doubt that the three factors make the systems more complex, and finding the stability conditions is quite a challenging task. Motivated by the above discussions, in this paper we will establish the stability criteria for hybrid impulsive and switching stochastic neural networks. The model is written as follows:

$$
\begin{aligned}
\mathrm{d} x(t)= & {\left[A_{\delta(k)} x(t)+f_{\delta(k)}(t, x(t-\tau))\right] \mathrm{d} t } \\
& +g_{\delta(k)}(t, x(t), x(t-\tau)) \mathrm{d} \omega(t), \\
t \in\left[t_{k-1}, t_{k}\right), & \\
\Delta x\left(t_{k}\right)= & B_{k}\left(t_{k}^{-}, x\left(t_{k}^{-}\right)\right), \quad t=t_{k}, \\
x\left(t_{0}, \delta\left(t_{0}\right)\right)= & \xi\left(t_{0}+s\right), \quad s \in[-\tau, 0), \\
x\left(t_{k}\right)= & Z_{\delta(k)},
\end{aligned}
$$

where $t \in R^{+}, x \in R^{n}$ is the state variable, $t_{0} \geq 0$ is the initial time, $\delta(t): R^{+} \rightarrow I, I=\{1,2, \ldots, m\}, R^{+}$is the positive real number set, and the time sequence $t_{k}$ satisfies $0 \leq t_{0}<t_{1}<$ $\cdots<t_{k}<\cdots, \lim _{k \rightarrow \infty} t_{k}=\infty$.

In fact, if we do not consider the stochastic factor of systems (3) (i.e., $g_{\delta(k)}(t, x(t), x(t-\tau))=0$ ), the systems reduce to ordinary differential equation systems. For example, the model become a hybrid impulsive and switching NN model without stochastic effects (see $[13-16,26])$. If $f_{\delta(k)}(t, x(t-$ $\tau))=0$, the model is a linear switching system considered in $[3,4]$. If $f_{\delta(k)}(t, x(t-\tau))=C_{\delta(k)} x(t-\tau)$, the model is described in [5]. In addition, systems (3) reduce to an impulsive stochastic differential equation without switching in [27], when $\delta(k)$ is a constant function and $g_{\delta(k)}(t, x(t), x(t-$ $\tau)) \neq 0$. If $B_{k}=0$, the system is a stochastic differential switching system without impulsive effects. By the discussion above, we draw a conclusion that many existing systems are included in systems (3).

This paper is organized as follows: The hybrid impulsive and switching stochastic neural networks and some kinds of stability are defined in Section 2. Section 3 constructs a type of switching signal and establishes several criteria for global asymptotical and exponential stability of a kind of impulsive stochastic differential switching systems with time delay. In Section 4, a numerical example is given to illustrate the theoretical results. Finally, Section 5 contains some conclusions.

Notation. Unless otherwise specified, we employ the definition as follows: Let $\left(\Omega, F,\left\{F_{t}\right\}_{t \geq 0}, P\right)$ be a complete probability space with filtration $\left\{F_{t}\right\}_{t} \geq 0$ satisfying the usual conditions (i.e., $F_{0}$ contains all $p$-null sets and $F_{t}$ is right continuous) and $\|\cdot\|$ denote the Euclidean norm of $R^{n}$. Let $E[\cdot]$ be the expectation operator with respect to the probability space. $\omega(t)=\left(\omega_{1}(t), \ldots, \omega_{n}(t)\right)^{T}$ represents $n$-dimensional Brownian with $E(\mathrm{~d} \omega(t))=0, E\left((\mathrm{~d} \omega(t))^{2}\right)=\mathrm{d} t$.

\section{Preliminaries}

To begin with, we introduce some conditions, basic definitions, and lemmas. Throughout this paper, it is assumed that the solution of systems (3) is unique and existing and satisfies the following conditions in any bounded interval $0<t_{k}-$ $t_{k-1} \leq T$ :

(C1) $B_{k}\left(t_{k}^{-}, x\left(t_{k}^{-}\right)\right): R^{+} \times R^{n} \rightarrow R^{n}(k \geq 1)$ are all continuous functions, and $B_{1}(t, 0) \equiv 0$ for any $t \in R^{+}$.

(C2) There exist nonnegative constant sequences $\left\{E_{\delta(k)}\right\}$ and $\left\{F_{\delta(k)}\right\}$ such that

(i) $\left\|f_{\delta(k)}(t, x(t))\right\|+\left\|g_{\delta(k)}(t, x(t))\right\| \leq E_{\delta(k)}(1+$ $\|x(t)\|)$

(ii) $\left\|f_{\delta(k)}(t, x)-f_{\delta(k)}(t, y)\right\|+\| g_{\delta(k)}(t, x)-$ $g_{\delta(k)}(t, y) \| \leq F_{\delta(k)}(\|x-y\|)$.

(C3) Let $x\left(t_{k}\right)=Z_{\delta(k)}$ be a random variable which is independent of the $\delta$-algebra $F_{\infty}^{(m)}$ generated by $\omega(\cdot)(\omega(\cdot)$ is an $m$-dimensional normal Brownian motion), and $E\left[\left\|Z_{\delta(k)}\right\|^{2}\right] \leq M<\infty$.

Let $T_{i}\left(t_{0}, t\right)=\sum_{k \in \Lambda}\left[\min \left(t, t_{k}\right)-t_{k-1}\right]$ denote the working time of the $i$ th subsystem during the interval $\left[t_{0}, t\right], \mu\left(T_{i}\left(t_{0}, t\right)\right)$ denote the Lebesgue measure of the set $T_{i}\left(t_{0}, t\right), k_{0}=$ $\min \left\{k \in Z, t_{k} \geq k\right\}, \Lambda=\left\{1,2, \ldots, k_{0}\right\} \cap\{k \in Z, \delta(k)=i\}$. Then systems (3) can be written as

$$
\begin{aligned}
\mathrm{d} x(s)= & {\left[A_{i} x(s)+f_{i}(s, x(s-\tau))\right] \mathrm{d} s } \\
& +g_{i}(s, x(s), x(s-\tau)) \mathrm{d} \omega(s),
\end{aligned}
$$




$$
\begin{aligned}
\Delta x(s) & =B_{k}\left(s^{-}, x\left(s^{-}\right)\right), \quad s=t_{k}, \\
x\left(t_{0}, \delta\left(t_{0}\right)\right) & =\xi\left(t_{0}+l\right), \quad l \in[-\tau, 0), \\
x\left(s_{k}\right) & =Z_{i},
\end{aligned}
$$

where $i \in I$ and $\bigcup_{i=1}^{N} T_{i}\left(t_{0}, t\right)=\left[t_{0}, t\right], f_{i}(0, x(0))=$ $g_{i}(0, x(0))=0$. Let $\Gamma=\left\{t_{i}: 0 \leq t_{1}<t_{2}<\cdots<t_{i}<\cdots\right\}$ and $R_{\delta}=\{x \in R: x \geq \delta\}$, where $\delta \in R$ is a given constant, and $C^{2,1}\left(R^{n} \times R_{\delta} \times I ; R^{+}\right)$denote a family of all nonnegative functions $V(x(t), t, i)$ on $R^{n} \times R_{\delta} \times I$ that are twice continuously differentiable in $x$ and once in $t$. If $V(x(t), t, i) \in$ $C^{2,1}\left(R^{n} \times R_{\delta} \times I ; R^{+}\right)$, define an operator $L$ associated with systems (3) and (4) from $R^{n} \times R_{\delta} \times I$ to $R$ by

$$
\begin{aligned}
& L V(x(t), t, i)=V_{t}(x(t), t, i)+V_{i}(x(t), t, i)\left[A_{i} x(t)\right. \\
& \left.\quad+f_{i}(t, x(t-\tau))\right]+\frac{1}{2} \operatorname{trace}\left[g_{i}^{T}(t, x(t), x(t-\tau))\right. \\
& \left.\quad \cdot V_{x x}(x(t), t, i) g_{i}(t, x(t), x(t-\tau))\right]
\end{aligned}
$$

where

$$
\begin{aligned}
& V_{t}(x(t), t, i)=\frac{\partial V(x(t), t, i)}{\partial t}, \\
& V_{x x}(x(t), t, i)=\left(\frac{\partial^{2} V(x(t), t, i)}{\partial x_{i} \partial x_{j}}\right)_{n \times n}, \\
& V_{x}(x(t), t, i)=\left(\frac{\partial V(x(t), t, i)}{\partial x_{1}}, \frac{\partial V(x(t), t, i)}{\partial x_{2}}, \ldots,\right. \\
& \left.\frac{\partial V(x(t), t, i)}{\partial x_{n}}\right) .
\end{aligned}
$$

Definition 1. (i) Asymptotically stable in mean square: the trivial solution of systems (3) and (4) is said to be asymptotically stable in mean square, for $\varepsilon>0$, if there exists $\delta=\delta\left(\varepsilon, t_{0}\right)>0$ such that

$$
\begin{aligned}
E\left(\left\|x\left(t_{0}\right)\right\|^{2}\right) & \leq \delta, \\
\lim _{t \rightarrow \infty} E\left(\|x(t)\|^{2}\right) & =0, \quad t \geq t_{0} .
\end{aligned}
$$

(ii) Exponentially stable in mean square: the trivial solution of systems (3) and (4) is said to be mean square exponentially stable if there exists positive constant $\alpha>0$ such that

$$
E\left(\|x(t)\|^{2}\right) \leq E\left(\left\|x\left(t_{0}\right)\right\|^{2}\right) e^{-\alpha\left(t-t_{0}\right)}, \quad t>t_{0} \geq 0 .
$$

Definition 2. $D^{+} f(x)=\lim _{\Delta x \rightarrow 0^{+}}((f(x+\Delta x)-f(x)) / \Delta x)$ if $f(x)$ is differentiable at its right side.

Furthermore, we introduce the following lemmas.
Lemma 3 (a generalized Halanay's inequality [33]). Let $\omega(t)$ be a nonnegative function defined on the interval $\left[t_{0}-\tau, \infty\right)$ and be continuous on the interval $\left[t_{0}, \infty\right)$. Assume that

$$
\dot{\omega}(t) \leq-a(t) \omega(t)+b(t) \omega(t-\tau), \quad t \geq t_{0}
$$

where $a(t)$ and $b(t)$ are nonnegative functions satisfying $a(t)>$ $b(t)$. Then

$$
\omega(t) \leq \bar{\omega}_{0} \exp \left(-\int_{t_{0}}^{t} \lambda \mathrm{d} s\right)
$$

where $\bar{\omega}=\sup _{t_{0}-\tau \leq \theta \leq t_{0}} \omega(\theta)$ and $\lambda>0$ satisfying

$$
\lambda-a(t)+b(t) e^{\lambda \tau}=0 .
$$

Lemma 4. For any $t_{1}<t_{2}$, if $V(x, t, i) \in C^{2,1}\left(R^{n} \times R_{\delta} \times S ; R^{+}\right)$, then

$$
\begin{aligned}
E V\left(x\left(t_{2}\right), t_{2}, \delta\left(t_{2}\right)\right)= & E V\left(x\left(t_{1}\right), t_{1}, \delta\left(t_{1}\right)\right) \\
& +E \int_{t_{1}}^{t_{2}} L V(x(s), s, \delta(s)) \mathrm{d} s .
\end{aligned}
$$

Lemma 5. For any $x, y \in R^{n}$, there exist nonnegative functions $h_{i}(t)$, such that

$$
f_{i}^{T}(t, x) y \leq h_{i}(t) x^{T} y, \quad i \in\{1,2, \ldots, N\}, t \leq t_{0} .
$$

Lemma 6. For all $i \in I$, if $a(t) \geq \alpha(t)>\alpha^{0}(t)>v(t) \geq b(t)$, then there exist $\lambda$ and $\lambda^{0}$ such that $\bar{\lambda} \geq \lambda>\lambda^{0}>0$ satisfying $\lambda-\alpha(t)+v(t) e^{\lambda \tau}=0$ and $\lambda^{0}-\alpha^{0}(t)+v(t) e^{\lambda^{0} \tau}=0$, where $a(t)$, $b(t)$, and $\bar{\lambda}$ are defined in Lemma 3.

Lemma 7. Let $\omega(t)$ be a nonnegative function defined on the interval $\left[t_{0}-\tau, \infty\right)$ and be continuous on the interval $\left[t_{0}, \infty\right)$. Assume that

$$
\dot{\omega}(t) \leq-a \omega(t)+b \omega(t-\tau), \quad t \geq t_{0}
$$

where $a$ and $b$ are nonnegative constants satisfying $a>b$. Then

$$
\omega(t) \leq \bar{\omega}_{0} \exp \left(-\int_{t_{0}}^{t} \lambda \mathrm{d} s\right)
$$

where $\bar{\omega}=\sup _{t_{0}-\tau \leq \theta \leq t_{0}} \omega(\theta)$ and $\lambda>0$ satisfying

$$
\lambda-a+b e^{\lambda \tau}=0
$$

\section{Stability Analysis}

Before stating our main results, we need to construct switching signals for systems (3) and (4). In fact, if there exist positive-definite and symmetric matrices $P_{i}$, such that

$$
Q=\sum_{i=1}^{N} \alpha_{i}\left(A_{i}^{T} P_{i}^{T}+P_{i} A_{i}\right),
$$


where $Q$ is a negative matrix, $\alpha_{i} \in[0,1]$, and $\sum_{i=1}^{N} \alpha_{i}=1$, then the solution of system (4) is stable. Thus, for any $x \neq 0$, we obtain that

$$
\sum_{i=1}^{N} \alpha_{i} x^{T}\left(A_{i}^{T} P_{i}^{T}+P_{i} A_{i}\right) x=x^{T} Q x \leq 0 .
$$

Note $\alpha_{i} \in[0,1]$. Based on formula (18), we know that there at least exist $\alpha_{i}, i \in\{1,2, \ldots, N\}$ such that

$$
\alpha_{i} x^{T}\left(A_{i}^{T} P_{i}^{T}+P_{i} A_{i}\right) x<0 .
$$

We construct switching signal area $\Omega_{i}$ as follows:

$$
\Omega_{i}=\left\{x \in R^{n}: x^{T}\left(A_{i}^{T} P_{i}^{T}+P_{i} A_{i}\right) x \leq x^{T} Q x\right\} .
$$

Theorem 8. Assume that $\Omega_{i}$ has the same form as formula (20), then

$$
R^{n}=\bigcup_{i=1}^{N} \Omega_{i}
$$

Proof. Suppose that formal (21) does not hold, then there exists a set $D \subset R^{n}$, such that $D=R^{n} \backslash \bigcup_{i=1}^{N} \Omega_{i}$. Thus, for any $i \in\{1,2, \ldots, N\}, x \in D$, we obtain that

$$
x^{T}\left(A_{i}^{T} P_{i}^{T}+P_{i} A_{i}\right) x>x^{T} Q x .
$$

For any $\alpha_{i} \in[0,1], \sum_{i=1}^{N} \alpha_{i}=1$, we have

$$
\sum_{i=1}^{N} \alpha_{i} x^{T}\left(A_{i}^{T} P_{i}^{T}+P_{i} A_{i}\right) x>\sum_{i=1}^{N} \alpha_{i} x^{T} Q x=x^{T} Q x .
$$

However, based on formula (18), formula (23) is impossible to hold. This completes the proof.

Based on Theorem 8 , we can find $N$ subdomain of $R^{n}$ for systems (3) and (4). Several new subdomains $\bar{\Omega}_{i}\left(\bar{\Omega}_{i} \subset\right.$ $\left.\bar{\Omega}_{j}, i \leq j, i, j=1,2, \ldots, N\right)$ are structured as follows:

$$
\bar{\Omega}_{i}=\bigcup_{j=1}^{i} \Omega_{j}, \quad i=1,2, \ldots, N
$$

Theorem 9. Assume that systems (3) and (4) satisfy the following conditions:

(C4) There exist symmetric and positive-definite matrices $P_{i}$, such that $Q=\sum_{i=1}^{N} \alpha_{i}\left(A_{i}^{T} P_{i}^{T}+P_{i} A_{i}\right)$, where $\alpha_{i} \in[0,1]$, $\sum_{i=1}^{N} \alpha_{i}=1$, and $Q$ is negative definite matrix.

(C5) For any $x, y \in R^{n}$, there exist continuous functions $h_{i}(t) \geq 0$ such that

$$
f_{i}^{T}(t, y) y \leq h_{i}(t) x^{T} y, \quad i \in\{1,2, \ldots, N\}, t \geq t_{0} .
$$

(C6) $\operatorname{trace}\left[g_{i}^{T}(t, x(t, x(t-\tau))) g_{i}(t, x(t), x(t-\tau))\right] \leq$ $\lambda_{i}^{(1)}\|x(t)\|^{2}+\lambda_{i}^{(2)}\|x(t-\tau)\|^{2}$.
(C7) $\lambda_{\text {max }}\left(P_{i}^{-1}\right)\left(\lambda_{\max }(Q)+\lambda_{i}^{(1)} \lambda_{\max }\left(P_{i}\right) \quad+\right.$ $\left.\left(\lambda_{\max }\left(P_{i}^{T} S_{i} P_{i}\right)\right) h_{i}(t)\right)+\alpha_{i}(t)<0, t \geq t_{0}, i \in$ $\{1,2, \ldots, N\}$.

(C8) $\sum_{j=1}^{k-1}\left(\ln \left(\lambda_{\text {max }}\left(P_{i}\right) / \lambda_{\text {min }}\left(P_{i}\right)\right)+2 \ln \left(1 / \lambda_{\text {min }}\left(E-B_{j}\right)\right)\right)-$ $\sum_{i=1}^{N}\left(\lambda_{i}\left(t_{j}-t_{j-1}\right)\right) \leq \varphi\left(t_{0}, t\right), t \in\left[t_{k-1}, t_{k}\right)$, where $\lambda_{i}$ is the unique positive root of the equation $-\alpha_{i}(t)+$ $\nu_{i}(t) e^{\lambda_{i} \tau}+\lambda_{i}=0$.

Then, it follows that $\varphi\left(t_{0}, t\right) \leq-c\left(t-t_{0}\right), c>0, t \geq t_{0}$, implies that trivial solution of systems (3) and (4) is globally exponentially stable in mean square and $\lim _{t \rightarrow \infty} \varphi\left(t_{0}, t\right)=-\infty$ implies that the trivial solution of systems (3) and (4) is globally asymptotically stable in mean square.

Proof. Suppose

$$
\begin{aligned}
\Omega_{i}=\left\{x \in R^{n}: x^{T}\left(A_{i}^{T} P^{T}+P_{i} A_{i}\right) x\right. & \left.\leq x^{T} Q x\right\}, \\
& i \in\{1,2, \ldots, N\},
\end{aligned}
$$

where $P_{i}$ are positive-definite matrices. We have $R^{n}=\bigcup_{i=1}^{N} \Omega_{i}$ and construct the $\bar{\Omega}_{i}$ as formula (24). Construct the switching Lyapunov function by

$$
V_{i}(t)=x^{T}(t) P_{i} x(t), \quad t \in\left[t_{k-1}, t_{k}\right)
$$

and set the switching rule as follows:

$$
\delta=N-i+1, \quad i \in\{1,2, \ldots, N\}
$$

where $x(t) \in \bar{\Omega}_{N} \backslash \bar{\Omega}_{N-i}$ and there at least exists $t^{*}$ such that $x\left(t^{*}\right) \in \bar{\Omega}_{N-i-1}, \bar{\Omega}_{0} \triangleq \emptyset$. When $x(t) \in \bar{\Omega}_{N} \backslash \bar{\Omega}_{i-1}, t \in\left[t_{i-1}, t_{i}\right)$, there exists $t^{*}$ such that $x\left(t^{*}\right) \in \bar{\Omega}_{i}$. Some $x(t)$ have access to $\bar{\Omega}_{i} \backslash \bar{\Omega}_{i-1}$ and switching signal $\delta=i$ under the impulse effects. Using Itô's formula in (27) yields

$$
\mathrm{d} V_{i}(t)=L V_{i}(t) \mathrm{d} t+g_{i}(t, x(t)) \frac{\partial V_{i}(t)}{\partial t} \mathrm{~d} \omega(t) .
$$

Based on formulas (5) and (6), we obtain that

$$
\begin{aligned}
& L V_{i}(t)=x^{T}(t) P_{i}\left[A_{i} x(t)+f_{i}(t, x(t-\tau))\right] \\
& \quad+\left[A_{i} x(t)+f_{i}(t, x(t-\tau))\right]^{T} P_{i} x(t) \\
& \quad+\operatorname{trace}\left[g_{i}^{T}(t, x(t), x(t-\tau))\right. \\
& \left.\cdot g_{i}(t, x(t), x(t-\tau))\right] .
\end{aligned}
$$

Thus

$$
\begin{gathered}
L V_{i}(t)=x^{T}(t) A_{i}^{T} P_{i} x(t)+2 f_{i}^{T}(t, x(t-\tau)) P_{i} x(t) \\
+x^{T} P_{i} A_{i} x(t)+\frac{1}{2} \operatorname{trace}\left[g_{i}^{T}(t, x(t), x(t-\tau))\right. \\
\left.\cdot P_{i} g_{i}(t, x(t), x(t-\tau))\right] \leqslant x^{T}(t)\left(A_{i}^{T} P_{i}+P_{i} A_{i}\right)
\end{gathered}
$$




$$
\begin{aligned}
& \cdot x(t)+2 h_{i}(t) x^{T}(t-\tau) P_{i} x(t)+\lambda_{i}^{(1)} x^{T}(t) P_{i} x(t) \\
& +\lambda_{i}^{(2)} x^{T}(t-\tau) P_{i} x(t-\tau) \leqslant x^{T}(t)\left(A_{i}^{T} P_{i}+P_{i} A_{i}\right) \\
& \cdot x(t)+h_{i}(t)\left(x^{T}(t-\tau) S_{i}^{-1} x(t-\tau)+x^{T}(t)\right. \\
& \left.\cdot P_{i}^{T} S_{i} P_{i} x(t)\right)+\lambda_{i}^{(1)} x^{T}(t) P_{i} x(t)+\lambda_{i}^{(2)} x^{T}(t-\tau) \\
& \cdot P_{i} x(t-\tau) \leqslant x^{T}(t)\left(\lambda_{\max }\left(Q_{i}\right)\right. \\
& \left.+\lambda_{\max }\left(P_{i}^{T} S_{i} P\right) h_{i}(t)+\lambda_{i}^{(1)} \lambda_{\max }\left(P_{i}\right)\right) x(t)+x^{T}(t \\
& -\tau)\left(S_{i}^{-1} h_{i}(t)+\lambda_{i}^{(2)} P_{i}\right) x(t-\tau) \leqslant \lambda_{\max }\left(P_{i}^{-1}\right) \\
& \cdot\left(\lambda_{\max }\left(Q_{i}\right)\right. \\
& \left.+\lambda_{\max }\left(P_{i}^{T} S_{i} P\right) h_{i}(t)+\lambda_{i}^{(1)} \lambda_{\max }\left(P_{i}\right)\right) x^{T}(t) P_{i} x(t) \\
& +\lambda_{\max }\left(\left(S_{i}^{-1} h_{i}(t)+\lambda_{i}^{(2)} P_{i}\right) P_{i}^{-1}\right) x^{T}(t-\tau) P_{i} x(t \\
& -\tau) \leqslant-\alpha_{i}(t) V_{i}(t)+v_{i}(t) V_{i}(t-\tau) .
\end{aligned}
$$

Applying Lemma 3 to (31) yields

$$
\begin{aligned}
E\left(V_{i}(t)\right) \leq E\left(V_{i}\left(t_{k-1}\right)\right) \exp \left(-\lambda_{i}\left(t_{k}-t_{k-1}\right)\right), & \\
t & \in\left[t_{k}, t_{k-1}\right),
\end{aligned}
$$

where $\lambda_{i}$ satisfies $-\alpha_{i}(t)+\nu_{i}(t) e^{\lambda_{i} \tau}+\lambda_{i}=0$. that

Considering the discrete part of system (4), we can obtain

$$
\begin{aligned}
& \lambda_{\min }\left(P_{i}\right) E\left[\|x(t)\|^{2}\right] \leqslant E\left[V_{i}(t)\right] \\
& \quad \leqslant \frac{1}{\lambda_{\min }^{2}\left(E-B_{j}\right)} E\left[V_{i}\left(t_{k-1}^{-}\right)\right] \exp \left(-\lambda_{i}\left(t_{k}-t_{k-1}\right)\right) .
\end{aligned}
$$

Thus

$$
\begin{aligned}
& E\left[\|x(t)\|^{2}\right] \leqslant \frac{1}{\lambda_{\min }^{2}\left(E-B_{j}\right)} \frac{\lambda_{\max }\left(P_{i}\right)}{\lambda_{\min }\left(P_{i}\right)} \\
& \cdot E\left[\left\|x\left(t_{k-1}^{-}\right)\right\|^{2}\right] \exp \left(-\lambda_{i}\left(t_{k}-t_{k-1}\right)\right) \\
& \vdots \\
& \leqslant \prod_{j=1}^{k-1}\left(\frac{1}{\lambda_{\min }^{2}\left(E-B_{j}\right)} \frac{\lambda_{\max }\left(P_{i}\right)}{\lambda_{\min }\left(P_{i}\right)}\right) \exp \left(-\sum_{i=1}^{N} \lambda_{i}\left(t_{j}\right.\right. \\
& \left.\left.-t_{j-1}\right)\right) E\left[\left\|x\left(t_{0}\right)\right\|^{2}\right]
\end{aligned}
$$

$$
\begin{aligned}
\leqslant & E\left[\left\|x\left(t_{0}\right)\right\|^{2}\right] \exp \left(\sum _ { j = 1 } ^ { k - 1 } \left(2 \ln \left(\frac{1}{\lambda_{\min }\left(E-B_{j}\right)}\right)\right.\right. \\
& \left.\left.+\ln \left(\frac{\lambda_{\max }\left(P_{i}\right)}{\lambda_{\min }\left(P_{i}\right)}\right)\right)-\sum_{i=1}^{N} \lambda_{i}\left(t_{j}-t_{j-1}\right)\right) \\
\leqslant & E\left[\left\|x\left(t_{0}\right)\right\|^{2}\right] e^{\varphi\left(t_{0}, t\right)}, \quad t \in\left[t_{k-1}, t_{k}\right) .
\end{aligned}
$$

When $\varphi\left(t_{0}, t\right) \leq-c\left(t-t_{0}\right), c>0, t \geq t_{0}$, the trivial solution of systems (3) and (4) is globally asymptotically exponentially stable in mean square. When $\lim _{t \rightarrow \infty} \varphi\left(t_{0}, t\right)=$ $-\infty$, the trivial solution of systems (3) and (4) is globally asymptotically stable.

Remark 10. Theorem 8 gives general criteria for the asymptotic and exponential stability of systems (3) and (4) consisting of stable and/or unstable modes. If $g(t, x(t), x(t-\tau)) \equiv$ $0, t \in\left[t_{0},+\infty\right)$, the stochastic part has been missed. That is to say, the systems have become hybrid impulse and switching systems with delay, which have been discussed in [7-16]. If $g(t, x(t), x(t-\tau)) \equiv 0, t \in\left[t_{0},+\infty\right)$ and $\tau=0$, the models have been studied in [11]. Furthermore, switching systems without impulses have been investigated in [13].

Remark 11. If $h_{i}=L_{i}$ are all constants, then inequality (31) satisfies the Halanay inequality (Lemma 7). The inequality can be solved with relevance linear matrix inequality (LMI). The problem turns to solve an optimization problem as follows:

$$
\text { OP } \begin{cases}\min & \alpha_{i}, \\ \text { s.t. } & \text { relevance LMI holds, }\end{cases}
$$

where $\alpha_{i}$ satisfies inequality (33).

Remark 12. In Theorem 9, inequality of (C8) characterizes the switching effect $-\sum_{i=1}^{N} \lambda_{i}\left(t_{k}-t_{k-1}\right)$ and the impulse effect $\sum_{j=1}^{k-1}\left(2 \ln \left(1 / \lambda_{\min }\left(E-B_{j}\right)\right)+\ln \left(\lambda_{\max }\left(P_{i}\right) / \lambda_{\min }\left(P_{i}\right)\right)\right)$ in an aggregated form. There is no particular limitation to the switching sequence and switching subinterval.

Corollary 13. For systems (3) and (4), suppose conditions ((C1)-(C4)) hold; if there exists positive constant $\alpha$ such that

$$
\begin{aligned}
& \frac{2 \ln \left(1 / \lambda_{\min }\left(E-B_{k}\right)\right)+\ln \left(\lambda_{\max }\left(P_{i}\right) / \lambda_{\min }\left(P_{i}\right)\right)}{t_{k}-t_{k-1}} \\
& \leqslant \alpha, \quad k=1,2, \ldots,
\end{aligned}
$$

where $\lambda_{i} \geq \alpha>0$, then the trivial solution is globally exponentially stable. 
Proof. Let $\lambda=\min _{1 \leqslant i \leqslant N}\left\{\lambda_{i}\right\}$. Based on (31), we have

$$
\begin{aligned}
& \sum_{j=1}^{k-1}\left(2 \ln \left(\frac{1}{\lambda_{\min }\left(E-B_{j}\right)}\right)+\ln \left(\frac{\lambda_{\max }\left(P_{i}\right)}{\lambda_{\min }\left(P_{i}\right)}\right)\right) \\
& -\sum_{i=1}^{N} \lambda_{i}\left(t_{j}-t_{j-1}\right) \\
& \quad \sum_{j=1}^{k-1}\left(2 \ln \left(\frac{1}{\lambda_{\min }\left(E-B_{j}\right)}\right)+\ln \left(\frac{\lambda_{\max }\left(P_{i}\right)}{\lambda_{\min }\left(P_{i}\right)}\right)\right) \\
& -\lambda \sum_{j=1}^{k-1}\left(t_{j}-t_{j-1}\right) \leqslant \alpha \sum_{j=1}^{k-1}\left(t_{j}-t_{j-1}\right) \\
& -\lambda \sum_{j=1}^{k-1}\left(t_{j}-t_{j-1}\right) \leqslant-(\lambda-\alpha)\left(t-t_{0}\right), \\
& t \in\left[t_{k-1}, t_{k}\right), k=1,2, \ldots
\end{aligned}
$$

Then the trivial solution of systems (3) and (4) is globally exponentially stable. The proof of the corollary is completed.

Remark 14. Based on Corollary 13, the trivial solution of systems (4) is globally asymptotically exponentially stable if there exists $\alpha$ such that $0 \leqslant \alpha<\lambda_{i}$. Furthermore, the convergence rate of the trivial solution is $\left(\min \left(\lambda_{i}\right)-\alpha\right) / 2, i \in$ I.

Corollary 15. For all $i \in I$, if $\alpha_{i} \geqslant \alpha>\alpha^{0}>v \geqslant v_{i}$, the trivial solution of systems (3) and (4) is globally exponentially stable provided that

$$
\begin{aligned}
2 \ln \left(\frac{1}{\lambda_{\min }\left(E-B_{k}\right)}\right)+\ln \left(\frac{\lambda_{\max }\left(P_{i}\right)}{\lambda_{\text {min }}\left(P_{i}\right)}\right) \\
\quad-\lambda^{0}\left(t_{k}-t_{k-1}\right) \leqslant 0, \quad i \in I, k \in\{1,2, \ldots\},
\end{aligned}
$$

where $\lambda_{i} \geqslant \lambda>\lambda^{0}>0$ and $\lambda_{i}$ satisfies $-\alpha_{i}(t)+\nu_{i}(t) e^{\lambda_{i} \tau}+\lambda_{i}=$ 0 .

Proof. According to Lemma 6, there exist $\lambda$ and $\lambda^{0}$ such that $\lambda_{i} \geq \lambda>\lambda^{0}>0$ satisfying $-\alpha(t)+\eta(t) e^{\lambda \tau}+\lambda=0$ and $-\alpha^{0}(t)+\eta(t) e^{\lambda^{0} \tau}+\lambda^{0}=0$. Note that

$$
\begin{aligned}
& 2 \ln \left(\frac{1}{\lambda_{\min }\left(E-B_{k}\right)}\right)+\ln \left(\frac{\lambda_{\max }\left(P_{i}\right)}{\lambda_{\min }\left(P_{i}\right)}\right) \\
& \leqslant \lambda^{0}\left(t_{i}-t_{i-1}\right), \quad i \in I, k \in\{1,2, \ldots\} .
\end{aligned}
$$

Thus

$$
\begin{aligned}
& \sum_{j=1}^{k-1}\left(2 \ln \left(\frac{1}{\lambda_{\min }\left(E-B_{j}\right)}\right)+\ln \left(\frac{\lambda_{\max }\left(P_{i}\right)}{\lambda_{\min }\left(P_{i}\right)}\right)\right) \\
& \quad \leqslant \lambda^{0}\left(t-t_{0}\right) .
\end{aligned}
$$

Since $\lambda_{i} \geqslant \lambda>\lambda^{0}$, we have

$$
\begin{gathered}
\sum_{j=1}^{k-1}\left(2 \ln \left(\frac{1}{\lambda_{\min }\left(E-B_{j}\right)}\right)+\ln \left(\frac{\lambda_{\max }\left(P_{i}\right)}{\lambda_{\min }\left(P_{i}\right)}\right)\right) \\
-\sum_{i=1}^{N} \lambda_{i}\left(t_{j}-t_{j-1}\right) \leqslant-\left(\lambda-\lambda^{0}\right)\left(t-t_{0}\right) .
\end{gathered}
$$

Then the proof is completed.

Remark 16. It is easy to see that the expectation of trivial solution of systems (3) and (4) satisfies $E\|x(t)\| \leqslant$ $K e^{-\left(\left(\lambda-\lambda^{0}\right) / 2\right)\left(t-t_{0}\right)}$, and the convergence rate of the trivial solution is $\left(\lambda-\lambda^{0}\right) / 2$.

Theorem 17. For any $t \in\left[t_{k-1}, t_{k}\right)$, suppose that conditions ((C1)-(C4)) hold, there are $\left\|h_{i}(t)\right\| \leqslant L_{i}$, and

$$
\begin{gathered}
\sum_{j=1}^{k-1}\left(2 \ln \left(\frac{1}{\lambda_{\min }\left(E-B_{j}\right)}\right)+\ln \left(\frac{\lambda_{\text {max }}\left(P_{i}\right)}{\lambda_{\text {min }}\left(P_{i}\right)}\right)\right) \\
-\sum_{i=1}^{N} \lambda_{i}\left(t_{j}-t_{j-1}\right) \leqslant \varphi\left(t, t_{0}\right),
\end{gathered}
$$

where $\alpha_{i}, v_{i}$ satisfy $-\alpha_{i}+v_{i} e^{\lambda \tau}+\lambda=0$. Then $\lim _{t \rightarrow \infty} \varphi\left(t, t_{0}\right)=$ $-\infty$ implies that the trivial solution of systems (3) and (4) is globally asymptotically stable, and $\varphi\left(t, t_{0}\right)=-c\left(t-t_{0}\right), t \geqslant t_{0}$, $c>0$, which implies that the trivial solution of systems (3) and (4) is globally exponentially stable.

Proof. Reconstruct the switching Lyapunov function (27). Based on formula (30) and Lemma 7, we obtain that

$$
\begin{aligned}
& L V_{i}(t) \leqslant\left(\lambda_{\max }\left(Q_{i} P_{i}^{-1}\right)+h_{i}(t) \lambda_{\max }\left(P_{i}^{T} S_{i}\right)+\lambda_{i}^{(1)}\right) \\
& \cdot x^{T}(t) P_{i} x(t)+\left[\lambda_{i}^{(2)}+h_{i}(t) \lambda_{\max }\left(S_{i}^{-1} P_{i}^{-1}\right)\right] \\
& \cdot x^{T}(t-\tau) P_{i} x(t-\tau) \\
& \quad \leqslant\left(\lambda_{\max }\left(Q_{i} P_{i}^{-1}\right)+L_{i} \lambda_{\max }\left(P_{i}^{T} S_{i}^{T}\right)+\lambda_{i}^{(1)}\right) V_{i}(t) \\
& \quad+\left(\lambda_{i}^{(2)}+L_{i} \lambda_{\max }\left(S_{i}^{-1} P_{i}^{-1}\right)\right) V_{i}(t-\tau) \leqslant-\alpha_{i} V_{i}(t) \\
& \quad+v_{i} V_{i}(t-\tau) .
\end{aligned}
$$

Thus

$$
\begin{array}{rl}
E V_{i}(t) \leqslant E V_{i}\left(t_{k-1}\right) \exp \left(-\lambda_{i}\left(t_{k}-t_{k-1}\right)\right) & \\
t & t \in\left[t_{k-1}, t_{k}\right) .
\end{array}
$$

Considering the discrete part of system (4), one obtains that

$$
\begin{aligned}
& \lambda_{\min }\left(P_{i}\right) E\left[\|x(t)\|^{2}\right] \leqslant E\left[V_{i}(t)\right] \\
& \quad \leqslant \frac{1}{\lambda_{\min }^{2}\left(E-B_{k}\right)} E\left[V_{i}\left(t_{k-1}^{-}\right)\right] \exp \left(-\lambda_{i}\left(t_{k}-t_{k-1}\right)\right) .
\end{aligned}
$$


Therefore

$$
\begin{aligned}
E[ & \left.\|x(t)\|^{2}\right] \leqslant \frac{1}{\lambda_{\min }^{2}\left(E-B_{k}\right)} \frac{\lambda_{\max }\left(P_{i}\right)}{\lambda_{\min }\left(P_{i}\right)} \\
\cdot E\left[\left\|x\left(t_{k-1}^{-}\right)\right\|^{2}\right] \exp \left(-\lambda_{i}\left(t_{k}-t_{k-1}\right)\right) & \vdots \\
\leqslant & \prod_{j=1}^{k-1}\left(\frac{1}{\lambda_{\min }^{2}\left(E-B_{j}\right)} \frac{\lambda_{\max }\left(P_{i}\right)}{\lambda_{\min }\left(P_{i}\right)}\right) \exp \left(\sum_{i=1}^{N}-\lambda_{i}\left(t_{j}\right.\right. \\
& \left.\left.-t_{j-1}\right)\right) E\left[\left\|x\left(t_{0}\right)\right\|^{2}\right] \\
\leqslant & E\left[\left\|x\left(t_{0}\right)\right\|^{2}\right] \exp \left(\sum _ { j = 1 } ^ { k - 1 } \left(2 \ln \left(\frac{1}{\lambda_{\min }\left(E-B_{j}\right)}\right)\right.\right. \\
& \left.\left.+\ln \left(\frac{\lambda_{\max }\left(P_{i}\right)}{\lambda_{\min }\left(P_{i}\right)}\right)\right)-\sum_{i=1}^{N} \lambda_{i}\left(t_{j}-t_{j-1}\right)\right) \\
\leqslant & K e^{\varphi\left(t_{0}, t\right)}, \quad t \in\left[t_{k-1}, t_{k}\right) .
\end{aligned}
$$

Then $\lim _{t \rightarrow \infty} \varphi\left(t, t_{0}\right)=-\infty$ implies that the trivial solution of system (3) and (4) is globally asymptotically stable, and $\varphi\left(t, t_{0}\right)=-c\left(t-t_{0}\right), t \geqslant t_{0}, c>0$, implies that the trivial solution of systems (3) and (4) is globally exponentially stable.

Remark 18. Similarly, inequality (43) can be solved by linear matrix inequality (LMI) with $\alpha_{i}$. The problem turns to solve an optimization problem.

Remark 19. In Theorem 17, a general criterion for asymptotic and exponential stability of stochastic systems (3) and (4) has been given. Inequality (41) includes the switching effect $-\sum_{i=1}^{N} \lambda_{i}\left(t_{k}-t_{k-1}\right)$ and the impulse effect $\sum_{j=1}^{k-1}\left(2 \ln \left(1 /\left(\lambda_{\text {min }}\left(E-B_{j}\right)\right)\right)+\ln \left(\lambda_{\text {max }}\left(P_{i}\right) / \lambda_{\text {min }}\left(P_{i}\right)\right)\right)$ in an aggregated form. In order to keep $\varphi\left(t, t_{0}\right) \rightarrow-\infty$ or $\varphi\left(t, t_{0}\right)=$ $-c\left(t-t_{0}\right)$, the impulse effect $\sum_{j=1}^{k-1}\left(2 \ln \left(1 / \lambda_{\min }\left(E-B_{j}\right)\right)+\right.$ $\left.\ln \left(\lambda_{\max }\left(P_{i}\right) / \lambda_{\min }\left(P_{i}\right)\right)\right)$ should be negative or bounded. Thus, we obtain the following corollaries.

Corollary 20. In Theorem 17, if impulse effect $2 \ln \left(1 / \lambda_{\text {min }}(E-\right.$ $\left.\left.B_{k}\right)\right)+\ln \left(\lambda_{\max }\left(P_{i}\right) / \lambda_{\min }\left(P_{i}\right)\right)<0$, then the trivial solution of stochastic systems (3) and (4) is globally exponentially stable.

Proof. Based on inequality (46), we have

$$
\begin{gathered}
\sum_{j=1}^{k-1}\left(2 \ln \left(\frac{1}{\lambda_{\min }\left(E-B_{j}\right)}\right)+\ln \left(\frac{\lambda_{\max }\left(P_{i}\right)}{\lambda_{\min }\left(P_{i}\right)}\right)\right) \\
-\sum_{i=1}^{N} \lambda_{i}\left(t_{j}-t_{j-1}\right) \leqslant-\sum_{i=1}^{N} \lambda_{i}\left(t_{j}-t_{j-1}\right)
\end{gathered}
$$

$$
\begin{aligned}
\leqslant-\min \left(\lambda_{i}\right) \sum_{j=1}^{k-1}\left(t_{j}-t_{j-1}\right) \leqslant-c\left(t-t_{0}\right) & \\
t & \in\left[t_{k-1}, t_{k}\right) .
\end{aligned}
$$

Let $\varphi\left(t, t_{0}\right)=-c\left(t-t_{0}\right), c>0$; the trivial solution of stochastic systems (3) and (4) is globally exponentially stable.

Corollary 21. In Theorem 17 , if impulse effect $\mid 2 \ln \left(1 / \lambda_{\text {min }}(E-\right.$ $\left.\left.B_{k}\right)\right)+\ln \left(\lambda_{\max }\left(P_{i}\right) / \lambda_{\text {min }}\left(P_{i}\right)\right) \mid<M(\sqrt{k+1}-\sqrt{k}) / \sqrt{k+1} \sqrt{k}$, where $M$ is a positive constant, then the trivial solution of stochastic systems (3) and (4) is globally asymptotically exponentially stable.

Proof. Similarly, we obtain that

$$
\begin{aligned}
& \sum_{j=1}^{k-1}\left(2 \ln \left(\frac{1}{\lambda_{\min }\left(E-B_{j}\right)}\right)+\ln \left(\frac{\lambda_{\max }\left(P_{i}\right)}{\lambda_{\min }\left(P_{i}\right)}\right)\right) \\
& -\sum_{i=1}^{N} \lambda_{i}\left(t_{j}-t_{j-1}\right) \leqslant \sum_{j=1}^{k-1} \frac{M(\sqrt{k+1}-\sqrt{k})}{\sqrt{k+1} \sqrt{k}} \\
& -\sum_{i=1}^{N} \lambda_{i}\left(t_{j}-t_{j-1}\right) \leqslant\left(1-\frac{1}{\sqrt{k}}\right) M \\
& -\min \left(\lambda_{i}\right) \sum_{j=1}^{k-1}\left(t_{j}-t_{j-1}\right) \leqslant M-c\left(t-t_{0}\right), \\
& \quad t \in\left[t_{k-1}, t_{k}\right) .
\end{aligned}
$$

Let $\varphi\left(t, t_{0}\right)=M-c\left(t-t_{0}\right), c>0$. The trivial solution of systems (3) and (4) is globally asymptotically stable.

\section{Numerical Simulation}

In this section, an example is presented to illustrate the main theoretical results.

Consider systems (4) as follows:

$$
\begin{aligned}
\mathrm{d} x(t)= & {\left[A_{1} x(t)+C_{1} f_{1}(t, x(t-\tau))\right] \mathrm{d} t } \\
& +g_{1}(t, x(t), x(t-\tau)) \mathrm{d} \omega(t), \\
& \quad t \in[k T,(k+\theta) T),
\end{aligned}
$$

$$
\begin{array}{r}
\begin{aligned}
& \Delta x(t)= x(t)-x\left(t^{-}\right)=B_{1} x(t), \\
& t_{k}=(k+\theta) T, k=1,2, \ldots, \\
& \mathrm{d} x(t)=\left[A_{2} x(t)+C_{2} f_{2}(t, x(t-\tau))\right] \mathrm{d} t \\
&+ g_{2}(t, x(t), x(t-\tau)) \mathrm{d} \omega(t), \\
& t \in[(k+\theta) T,(k+1) T), \\
& \Delta x(t)=x(t)-x\left(t^{-}\right)=B_{2} x(t), \\
& t_{k}=(k+1) T, k=1,2, \ldots,
\end{aligned}
\end{array}
$$


TABLE 1: Maximum allowed $\tau$.

\begin{tabular}{lcccc}
\hline Methods & Our method & Pu and Tan [1] & Xing-cheng and Wei [15] & Li et al. [26] \\
\hline$\tau$ & 1.20 & 1.08 & 1.13 & 1.16 \\
\hline
\end{tabular}
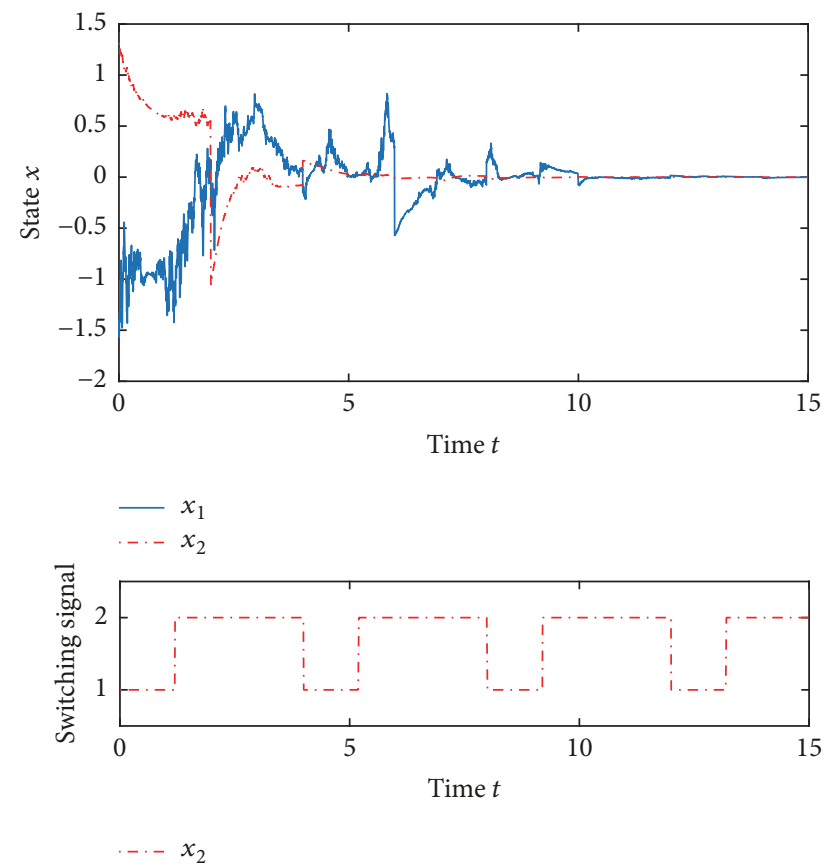

FIGURE 1: Trajectories of systems (49) with the initial value $x(s)=$ $[1.0,-1.48], s \in[-0.4,0]$.

where $T=4, \tau=1.2, B_{1}=1.9614, B_{2}=1.5437, A_{1}=A_{2}=$ $\left(\begin{array}{cc}-2 & 0 \\ 0 & -2\end{array}\right), C_{1}=\left(\begin{array}{cc}0.65 & -0.43 \\ 0.1 & 0.63\end{array}\right), C_{2}=\left(\begin{array}{cc}0.44 & 0.53 \\ -0.31 & 0.4\end{array}\right)$,

$$
f_{i}(t, x(t-\tau))=\sin (t)(|x+1|-|x-1|) .
$$

Let $D_{1}=\left(\begin{array}{cc}0.65 & -0.43 \\ 0.1 & 0.63\end{array}\right), D_{2}=\left(\begin{array}{cc}0.44 & 0.53 \\ -0.31 & 0.4\end{array}\right)$,

$$
\begin{aligned}
& g_{i}(t, x(t), x(t-\tau)) \\
& \quad=D_{i}(\sin (t) x(t)+\sin (t-\tau) x(t-\tau)), \\
& \quad i=1,2, t \geqslant t_{0} .
\end{aligned}
$$

The switching sequence is as follows: subsystem $1 \rightarrow$ subsystem $2 \rightarrow$ subsystem $1 \rightarrow \cdots$.

Construct the switching Lyapunov function as formula (27), where $x^{T}(t)=\left(x_{1}(t), x_{2}(t)\right)$ and

$$
P_{i}=\left(\begin{array}{ll}
2.4347 & 0.0324 \\
0.0324 & 2.7938
\end{array}\right) \text {. }
$$

Based on Corollary 13, we obtain that $\left(2 \ln \left(1 / \lambda_{\min }(E-\right.\right.$ $\left.\left.\left.B_{k}\right)\right)+\ln \left(\lambda_{\max }\left(P_{i}\right) / \lambda_{\min }\left(P_{i}\right)\right)\right)\left(1 /\left(t_{k}-t_{k-1}\right)\right)=0.1137 \leqslant$ $0.5, k=1,2, \ldots$. Thus, the trivial solution of systems (49) is exponentially stable, as shown in Figure 1.

The example results show that the stability of timevarying case can be guaranteed by our method. It is easy to see that model (49) and our conclusions can be degenerated into the general case if the $\alpha_{i}(t)$ and $v_{i}(t)$ are constants. It is the extension of $[1,15,26]$. Thus, compared with the existing results discussed in $[1,15,26]$, our results are less conservative. The tolerable delay upper bound of (49) is larger as shown in Table 1 .

\section{Conclusion}

In this paper, we have discussed the stability of hybrid impulsive and switching stochastic neural networks. A type of switching signals is constructed. Using switching Lyapunov functions and stochastic analysis techniques, several general criteria for the asymptotic and exponential stability analysis of the new model are established. To illustrate the advantage of the results, a numerical example is given.

\section{Conflicts of Interest}

The authors declare that they have no conflicts of interest.

\section{Acknowledgments}

The authors gratefully acknowledge the financial support from China Scholarship Council. This work is partially supported by the National Natural Science Foundation of China (Grants nos. 11761030, 11561022, and 61763009).

\section{References}

[1] X.-C. Pu and X.-G. Tan, "Stability of hybrid stochastic differential systems with switching and time delay," Stochastic Analysis and Applications, vol. 35, no. 3, pp. 569-585, 2017.

[2] J. Qi, C. Li, T. Huang, and W. Zhang, "Exponential Stability of Switched Time-varying Delayed Neural Networks with All Modes Being Unstable," Neural Processing Letters, vol. 43, no. 2, pp. 553-565, 2016.

[3] G. Conte, A. M. Perdon, and N. Otsuka, "The disturbance decoupling problem with stability for switching dynamical systems," Systems \& Control Letters, vol. 70, pp. 1-7, 2014.

[4] M. Wicks, P. Peleties, and R. DeCarlo, "Switched Controller Synthesis for the Quadratic Stabilisation of a Pair of Unstable Linear Systems," European Journal of Control, vol. 4, no. 2, pp. 140-147, 1998.

[5] S. Kim, S. A. Campbell, and X. Liu, "Stability of a class of linear switching systems with time delay," IEEE Transactions on Circuits and Systems I: Regular Papers, vol. 53, no. 2, pp. 384393, 2006.

[6] S. Ding, Z. Wang, Z. Huang, and H. Zhang, "Novel switching jumps dependent exponential synchronization criteria for memristor-based neural networks," Neural Processing Letters, vol. 45, no. 1, pp. 15-28, 2017.

[7] Y. Wang, X. Shi, Z. Zuo, M. Z. Q. Chen, and Y. Shao, "On finite-time stability for nonlinear impulsive switched systems," Nonlinear Analysis: Real World Applications, vol. 14, no. 1, pp. 807-814, 2013. 
[8] G. Zhai, B. Hu, K. Yasuda, and A. N. Michel, "Stability analysis of switched systems with stable and unstable subsystems: an average dwell time approach," International Journal of Systems Science, vol. 32, no. 8, pp. 1055-1061, 2001.

[9] Z. Li, H. Gao, and H. R. Karimi, "Stability analysis and H controller synthesis of discrete-time switched systems with time delay," Systems \& Control Letters 66, vol. 66, pp. 85-93, 2014.

[10] B. Hu and A. N. Michel, "Stability analysis of digital feedback control systems with time-varying sampling periods," Automatica, vol. 36, no. 6, pp. 897-905, 2000.

[11] S.-H. Lee and J.-T. Lim, "Stability analysis of switched systems with impulse effects," in Proceedings of the 1999 IEEE International Symposium on Intelligent Control - Intelligent Systems and Semiotics, pp. 79-83, September 1999.

[12] M. Kermani and A. Sakly, "Stability analysis of switched nonlinear time-delay systems," Systems Science \& Control Engineering: An Open Access Journal, vol. 2, no. 1, pp. 80-89, 2014.

[13] L. Zhang and P. Shi, "Stability, $l_{2}$-gain and asynchronous $H_{\infty}$ control of discrete-time switched systems with average dwell time," IEEE Transactions on Automatic Control, vol. 54, no. 9, pp. 2192-2199, 2009.

[14] M. S. Alwan and X. Liu, "On stability of linear and weakly nonlinear switched systems with time delay," Mathematical and Computer Modelling, vol. 48, no. 7-8, pp. 1150-1157, 2008.

[15] P. Xing-cheng and Y. Wei, "Stability of hybrid stochastic systems with time-delay," ISRN Mathematical Analysis, vol. 2014, 8 pages, 2014.

[16] X.-M. Sun, G. M. Dimirovski, J. Zhao, W. Wang, and S. S. Cyril, "Exponential stability for switched delay systems based on average dwell time technique and Lyapunov function method," in Proceedings of the 2006 American Control Conference, pp. 1539-1543, usa, June 2006.

[17] Y. Sun and J. Cao, "Stabilization of stochastic delayed neural networks with Markovian switching," Asian Journal of Control, vol. 10, no. 3, pp. 327-340, 2008.

[18] C. Huang and J. Cao, "On pth moment exponential stability of stochastic Cohen-Grossberg neural networks with timevarying delays," Neurocomputing, vol. 73, no. 4-6, pp. 986-990, 2010.

[19] H. Liu, L. Zhao, Z. Zhang, and Y. Ou, "Stochastic stability of Markovian jumping Hopfield neural networks with constant and distributed delays," Neurocomputing, vol. 72, no. 16-18, pp. 3669-3674, 2009.

[20] D. Li, X. Wang, and D. Xu, "Existence and global p-exponential stability of periodic solution for impulsive stochastic neural networks with delays," Nonlinear Analysis: Hybrid Systems, vol. 6, no. 3, pp. 847-858, 2012.

[21] H. Yang, Z. Wang, H. Shu, F. E. Alsaadi, and T. Hayat, "Almost sure Ho sliding mode control for nonlinear stochastic systems with Markovian switching and time-delays," Neurocomputing, vol. 175, pp. 392-400, 2015.

[22] W. Chen, S. Xu, B. Zhang, and Z. Qi, "Stability and stabilisation of neutral stochastic delay Markovian jump systems," IET Control Theory \& Applications, vol. 10, no. 15, pp. 1798-1807, 2016.

[23] G. Wang, Q. Zhang, and C. Yang, "Exponential stability of stochastic singular delay systems with general Markovian switchings,' International Journal of Robust and Nonlinear Control, vol. 25, no. 17, pp. 3478-3494, 2015.

[24] X. Wu, W. Zhang, and Y. Tang, " $p$ th moment stability of impulsive stochastic delay differential systems with Markovian switching," Communications in Nonlinear Science and Numerical Simulation, vol. 18, no. 7, pp. 1870-1879, 2013.

[25] T. Jiao, J. Lu, Y. Li, Y. Chu, and S. Xu, "Stability analysis of random systems with Markovian switching and its application," Journal of The Franklin Institute, vol. 353, no. 1, pp. 200-220, 2016.

[26] C. D. Li, G. Feng, and T. W. Huang, "On hybrid impulsive and switching neural networks," IEEE Transactions on Systems, Man, and Cybernetics, Part B: Cybernetics, vol. 38, no. 6, pp. 15491560, 2008.

[27] Z. G. Yang, D. Y. Xu, and L. Xiang, "Exponential p-stability of impulsive stochastic differential equations with delays," Physics Letters A, vol. 359, no. 2, pp. 129-137, 2006.

[28] E. Zhu and Q. Yuan, "Pth moment exponential stability of stochastic recurrent neural networks with Markovian switching," Neural Processing Letters, vol. 38, no. 3, pp. 487-500, 2013.

[29] S. Zhu, Y. Shen, and L. Liu, "Exponential stability of uncertain stochastic neural networks with markovian switching," Neural Processing Letters, vol. 32, no. 3, pp. 293-309, 2010.

[30] G. Wang, Q. Zhang, C. Yang, and C. Su, "Stability and stabilization of continuous-time stochastic Markovian jump systems with random switching signals," Journal of The Franklin Institute, vol. 353, no. 6, pp. 1339-1357, 2016.

[31] L. Pan and J. Cao, "Exponential stability of impulsive stochastic functional differential equations," Journal of Mathematical Analysis and Applications, vol. 382, no. 2, pp. 672-685, 2011.

[32] H. Zhang, Z. Wu, and Y. Xia, "Exponential stability of stochastic systems with hysteresis switching," Automatica, vol. 50, no. 2, pp. 599-606, 2014.

[33] L. Wen, Y. Yu, and W. Wang, "Generalized Halanay inequalities for dissipativity of Volterra functional differential equations," Journal of Mathematical Analysis and Applications, vol. 347, no. 1, pp. 169-178, 2008. 


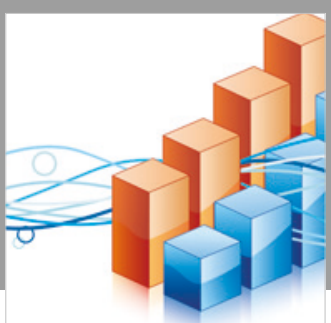

Advances in

Operations Research

\section{-n-m}
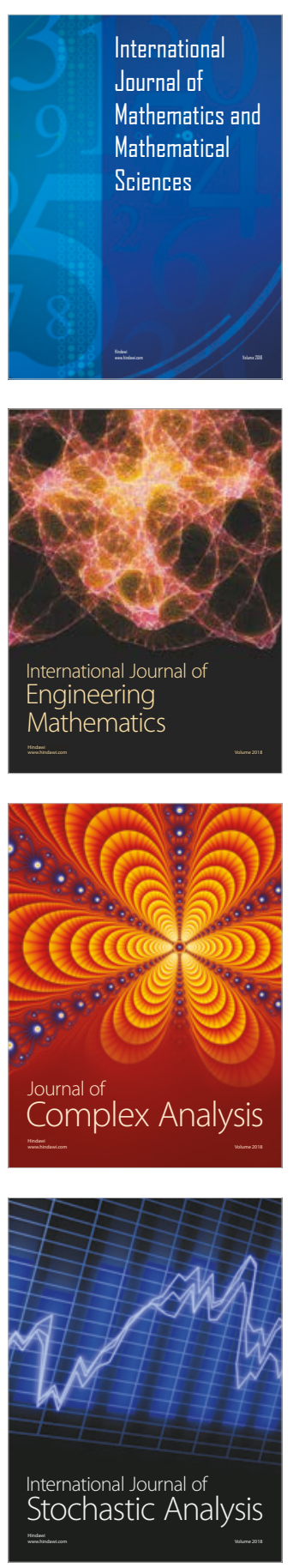
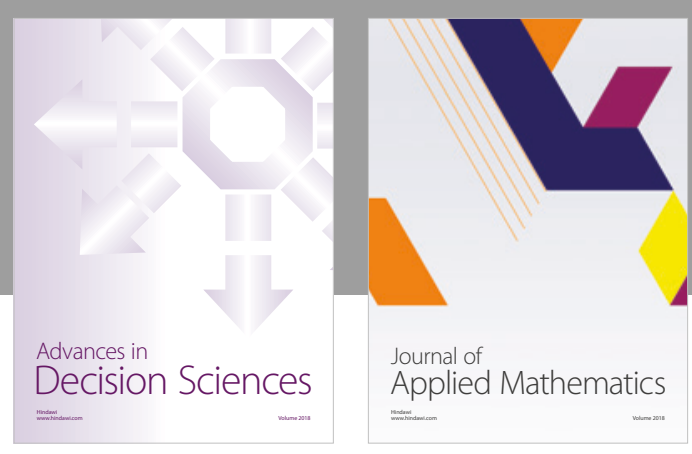

Journal of

Applied Mathematics
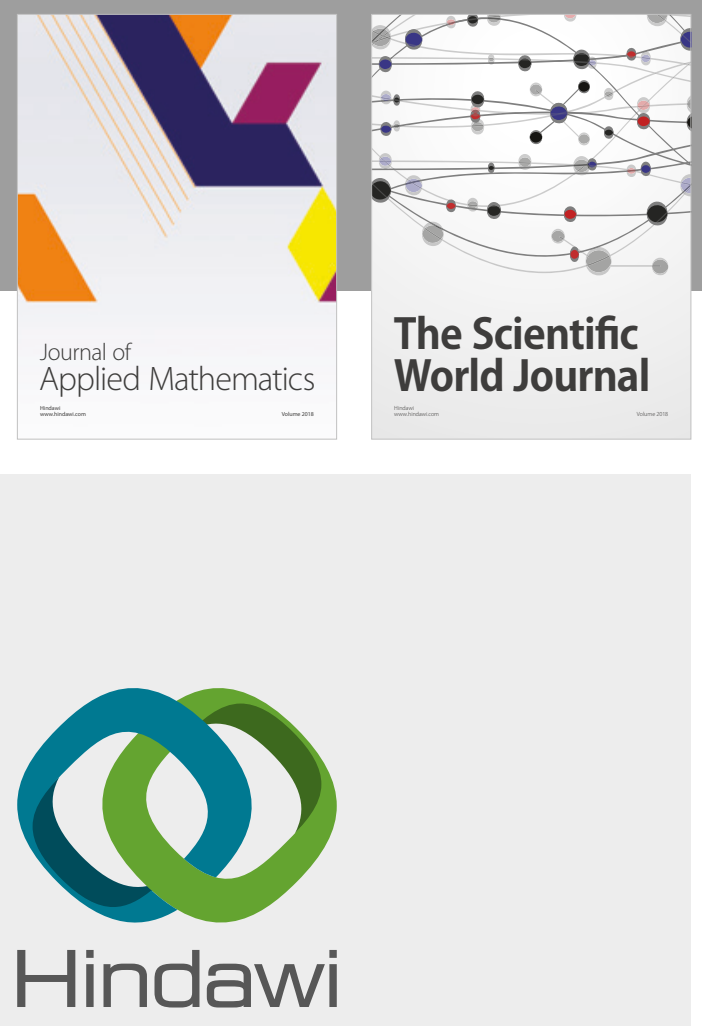

Submit your manuscripts at

www.hindawi.com

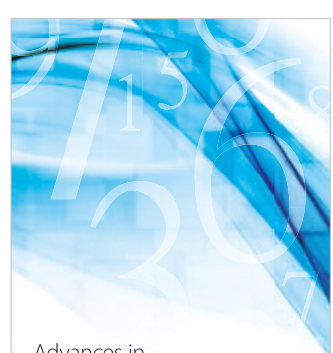

Advances in
Numerical Analysis
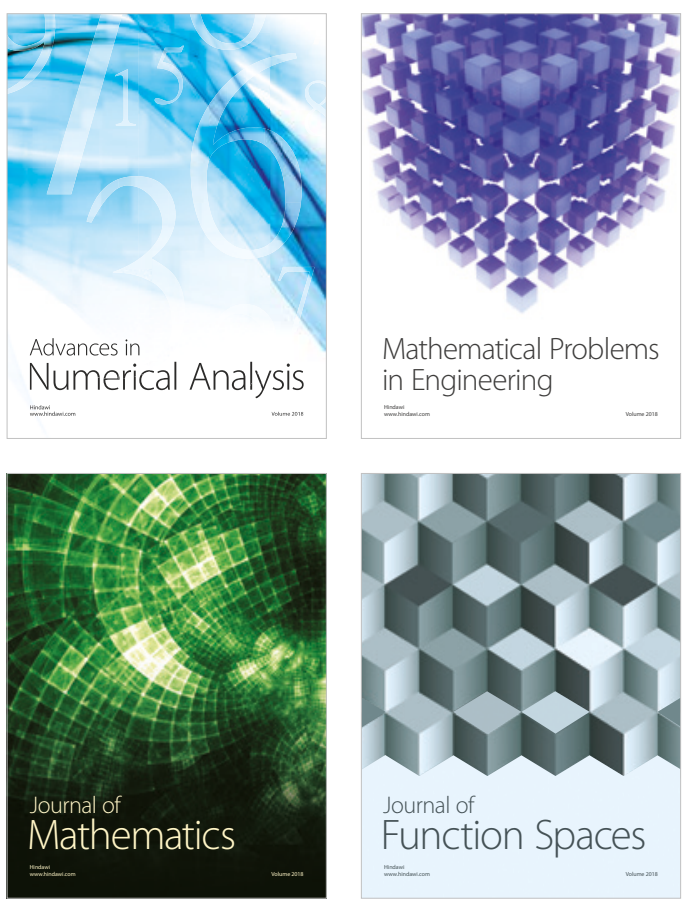

Mathematical Problems in Engineering

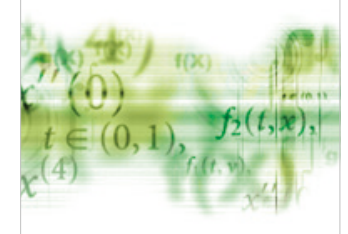

International Journal of

Differential Equations

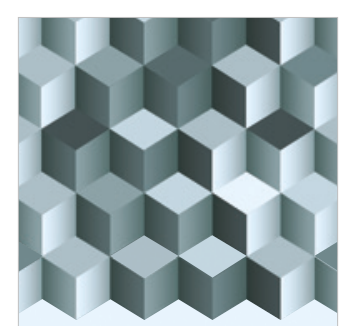

Journal of

Function Spaces

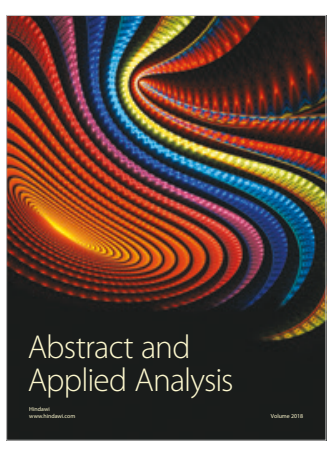

The Scientific

World Journal

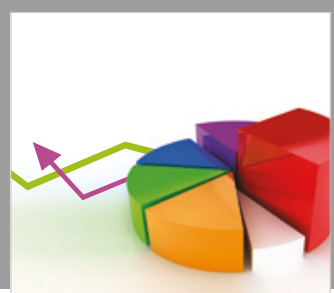

Journal of

Probability and Statistics
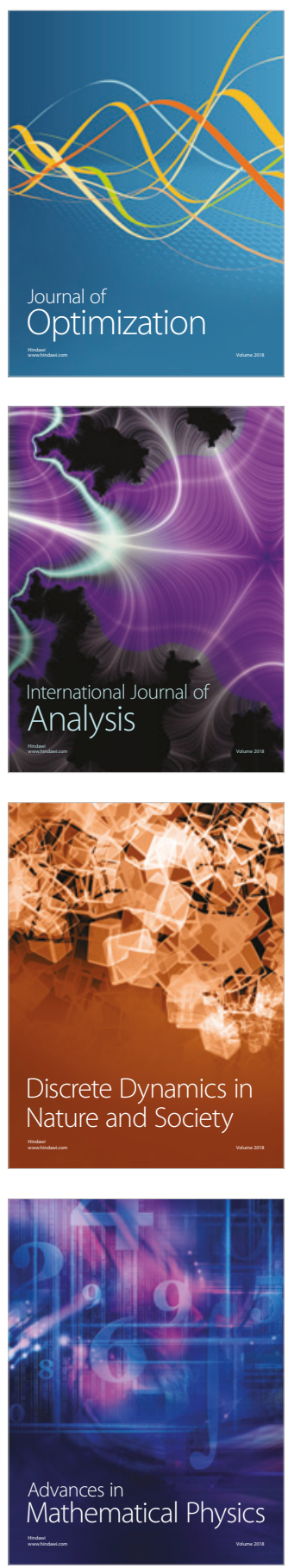